MENEZES JÚNIOR FOG; GONÇALVES PAS; VIEIRA NETO J. 2014. Produtividade da cebola em cultivo mínimo no sistema convencional e orgânico com biofertilizantes. Horticultura Brasileira 32: 475-481. DOI - http://dx.doi.org/10.1590/S0102-053620140000400017

\title{
Produtividade da cebola em cultivo mínimo no sistema convencional e orgânico com biofertilizantes
}

\author{
Francisco OG Menezes Júnior; Paulo AS Gonçalves; João Vieira Neto
}

Epagri, EE Ituporanga, C. Postal 121, 88400-000 Ituporanga-SC; franciscomenezes@epagri.sc.gov.br; pasg@epagri.sc.gov.br; joaoneto@epagri.sc.gov.br

\section{RESUMO}

As respostas em produtividade dos cultivos estão relacionadas, entre outros fatores, ao manejo de adubação e fitossanitário. O objetivo do experimento foi comparar a produtividade da cebola em sistema convencional e orgânico, em cultivo mínimo, relacionando-a a aspectos nutricionais e fitossanitários. Foram conduzidos dois experimentos, safras 2011 e 2012, em Cambissolo Háplico, em Ituporanga. Em 2011 foram aplicados os tratamentos: CONV (adubação mineral e pesticidas indicados para cultura da cebola); AM/CB [adubação mineral e calda bordalesa (CB 0,3\%)]; AO (adubação orgânica sem pesticidas); $\mathrm{AO} / \mathrm{CB}$ (adubação orgânica e $\mathrm{CB}$ 0,3\%); $\mathrm{AO} / \mathrm{CB} / \mathrm{BF}-3 \%$ [adubação orgânica, $\mathrm{CB}$ 0,3\% e pulverização de biofertilizante líquido à base de esterco bovino (PBEB a 3\%)]; $\mathrm{AO} / \mathrm{CB} / \mathrm{BF}-5 \%$ (adubação orgânica, $\mathrm{CB}$ 0,3\% e PBEB a 5\%); AO/CB/BF-10\% (adubação orgânica, $\mathrm{CB}$ 0,3\% e PBEB a 10\%). Em 2012, adicionou-se o tratamento AM (adubação mineral sem pesticidas). Utilizou-se a cultivar Empasc 352 Bola Precoce. Os transplantes foram realizados em 12/07/11 e 10/07/12, e as colheitas em 21/11/2011 e 09/11/2012. CONV foi $39 \%$ mais produtivo que a média dos tratamentos orgânicos com calda bordalesa e biofertilizantes. Medições indiretas de clorofila foram influenciadas pela fase do ciclo e estresse hídrico. Déficits hídricos reduziram a produtividade comercial total em 34\% em CONV e $43 \%$ em cultivos orgânicos. Biofertilizantes pulverizados não forneceram nutrientes, em especial $\mathrm{N}$, nas quantidades requeridas; não alteraram a qualidade nutricional, teor e extração de nutrientes pelos bulbos; e não reduziram a incidência de tripes e míldio na cebola.

Palavras-chave: Allium cepa, clorofila, nutrição, fitossanidade, qualidade nutricional.

\begin{abstract}
Yield of onion cultivated in conventional and organic minimum tillage systems with biofertilizers

The responses in yield of crops are related to fertilization and pest management. The objective of the experiment was to compare the yield of onion in organic and conventional system, under minimum tillage, related to nutritional and plant health aspects. Two experiments were carried out, in 2011 and 2012, over an Haplumbrept soil at Ituporanga, Santa Catarina state, Brazil. In 2011 we established the following treatments: CONV (conventional mineral fertilizer and pesticides), $\mathrm{AM} / \mathrm{CB}$ (mineral fertilizer and Bordeaux mixture (CB $0.3 \%$ )], $\mathrm{AO}$ (organic fertilizer without pest management); $\mathrm{AO} / \mathrm{CB}$ [organic fertilizer and $\mathrm{CB} 0.3 \%$ ); $\mathrm{AO} / \mathrm{CB} / \mathrm{BF}-3 \%$ [organic fertilizer, $\mathrm{CB} 0.3 \%$ and biofertilizer sprayed liquid of cow manure (PBEB $3 \%$ )]; $\mathrm{AO} / \mathrm{CB} / \mathrm{BF}-5 \%$ (organic fertilizer, $\mathrm{CB} 0.3 \%$ and $\mathrm{PBEB} 5 \%$ ); $\mathrm{AO} / \mathrm{CB} / \mathrm{BF}-10 \%$ (organic fertilizer, $\mathrm{CD} 0.3 \%$ and $\mathrm{PBEB} 10 \%$ ). In 2012, we included the AM treatment (mineral fertilizer without pest management). We used the Empasc 352 Bola Precoce cultivar. The seedlings were transplanted in July 21, 2011 and July 9, 2012, and the harvests were made in November 21, 2011 and September 9, 2012 , respectively. The CONV treatment was $39 \%$ more productive than the average organics with Bordeaux mixture and biofertilizers. Indirect measurements of chlorophyll were influenced by cycle phase and water stress. The water stress reduced the marketable yield in $43 \%$ on organic system and $34 \%$ in CONV. Biofertilizers did not provide nutrients, especially $\mathrm{N}$, in the required amounts by plants. Also, they did not alter the nutritional quality, content and nutrient uptake by the bulbs, and did not reduce the incidence of thrips and downy mildew in onions.
\end{abstract}

Keywords: Allium cepa, chlorophyll, nutrition, plant health, nutritional quality.

(Recebido para publicação em 7 de maio de 2013; aceito em 7 de agosto de 2014)

(Received on May 7, 2013; accepted on August 7, 2014)

\begin{abstract}
A cultura da cebola, em volume produzido e renda gerada, é a terceira espécie olerácea mais importante no Brasil (Bettoni, 2011). Por ser uma atividade tipicamente praticada por pequenos e médios agricultores, destaca-se do ponto de vista socioeconômico ao contribuir para a geração de renda, emprego e fixação do homem ao
\end{abstract}

meio rural. O estado de Santa Catarina é o principal produtor de bulbos, sendo responsável por $33 \%$ da safra nacional (IBGE, 2010). Estima-se que somente neste estado estejam envolvidas entre 8.000 e 10.000 famílias, cuja produção está $85 \%$ concentrada na região do Alto Vale do Itajaí. Não obstante, na maior parte das áreas cultivadas adota-se sis- temas de produção convencionais, nos quais há intensa mobilização do solo, uso excessivo de fertilizantes e agrotóxicos, técnicas responsáveis por impactos negativos ao ambiente e para homem.

Para amenizar tais problemas têm sido estudados sistemas alternativos de adubação e manejo fitossanitário. Entre esses estão o uso de fosfatos naturais, 
estercos curtidos e biofertilizantes, indicados como forma de manter o equilíbrio nutricional de plantas e menos predispostas à ocorrência de pragas e patógenos (Bettiol, 2001; Santos, 2001; Souza \& Alcântara, 2008).

Poucos estudos têm sido realizados visando correlacionar métodos de adubação e manejo fitossanitário ao teor e exportação de nutrientes, qualidade nutricional dos bulbos e produtividade nos sistemas de produção e cultivares em uso na região do Alto Vale do Itajaí-SC.

O objetivo deste experimento foi avaliar o efeito de diferentes manejos de adubação e fitossanitários na produtividade, teor e exportação de nutrientes, qualidade nutricional dos bulbos, incidência de míldio e tripes, e perda de massa fresca dos bulbos em pós-colheita da cultivar de cebola Empasc 352 Bola Precoce, em sistema de cultivo mínimo, nas condições do Alto Vale do Itajaí-SC.

\section{MATERIAL E MÉTODOS}

Dois experimentos foram conduzidos, em 2011 e 2012, na Epagri, Estação Experimental de Ituporanga (Epagri-EEItu), localizada no município de Ituporanga-SC $\left(27^{\circ} 38^{\prime} \mathrm{S}, 4^{\circ} 60^{\prime} \mathrm{O}\right.$, altitude $\left.475 \mathrm{~m}\right)$. Segundo a classificação de Köeppen, o clima local é do tipo $\mathrm{Cfa}$.

Durante os experimentos, registraram-se na Estação Meteorológica da Epagri-EEItu valores médios, em 2011 e 2012 , respectivamente, de: 82 e $80 \%$ de umidade relativa do ar; 10,1 e $11,9^{\circ} \mathrm{C}$ de temperatura mínima média do ar, 15,0 e $17,3^{\circ} \mathrm{C}$ de temperatura média do ar, e 21,9 e $22,6^{\circ} \mathrm{C}$ de temperatura máxima média do ar. Os dados mensais de precipitação do transplante à colheita dos bulbos de cada ano agrícola foram em 2011 e 2012, respectivamente: julho $=136,4$ e $155,0 \mathrm{~mm}$; agosto $=365,0 \mathrm{e}$ $18,3 \mathrm{~mm}$; setembro = 260,3 e 69,7 mm; outubro = 133,4 e 207,2 mm; novembro $=48,4$ e $15,1 \mathrm{~mm}$; total precipitado no período de 933,5 e 465,3 mm.

Avaliou-se a cv. Empasc 352 Bola Precoce. A semeadura foi realizada em 25/04/11 e 19/04/12; o transplante em $12 / 07 / 11$ e $10 / 07 / 12$, e a colheita em 21/11/2011 e 09/11/2012.

As mudas foram produzidas tendo por base os referenciais tecnológicos propostos pela Epagri (2000). A adubação dos canteiros constou de $0,5 \mathrm{~kg}$ de esterco de peru e $200 \mathrm{~g} / \mathrm{m}^{2}$ da formulação NPK 5-20-10. Nesta fase, os tratamentos fitossanitários foram realizados com fungicidas químicos registrados para a cultura.

O solo em que foi instalado o experimento é classificado como Cambissolo Háplico. No experimento de 2011 foram aplicados sete tratamentos: CONV (adubação mineral e pesticidas comerciais); $\mathrm{AM} / \mathrm{CB}$ (adubação mineral e calda bordalesa 0,3\%); AO (adubação orgânica sem tratamento fitossanitário); $\mathrm{AO} / \mathrm{CB}$ (adubação orgânica e calda bordalesa $0,3 \%$ ); $\mathrm{AO} / \mathrm{CB} / \mathrm{BF}-3 \%$ (adubação orgânica, calda bordalesa 0,3\%, complementada com pulverização de biofertilizante líquido à base de esterco bovino a 3\%); AO/CB/BF-5\% (adubação orgânica e calda bordalesa $0,3 \%$, complementada com pulverização de biofertilizante líquido à base de esterco bovino a 5\%); $\mathrm{AO} /$ CB/BF-10\% (adubação orgânica e calda bordalesa $0,3 \%$, complementada com pulverização de biofertilizante líquido à base de esterco bovino a 10\%). No experimento de 2012 adicionou-se aos tratamentos o tratamento AM (adubação mineral sem tratamento fitossanitário).

O delineamento experimental utilizado foi de blocos ao acaso, com quatro repetições. A área total de cada parcela experimental foi de 9,6 $\mathrm{m}^{2}(3,0 \times 3,2 \mathrm{~m})$, tendo como área útil $6,31 \mathrm{~m}^{2}$.

As análises de solo foram realizadas nos laboratórios da Epagri, no Centro de Pesquisa para Agricultura Familiar, Cepaf/Chapecó-SC, e da Estação Experimental de Ituporanga, credenciados pela ROLAS-RS/SC. As análises foram realizadas em amostras retiradas da profundidade de $0-20 \mathrm{~cm}$, apresentando os resultados, para 2011 e 2012, respectivamente: argila $=500$ e $485 \mathrm{~g} / \mathrm{dm}^{3} ; \mathrm{pH}\left(\mathrm{H}_{2} \mathrm{O}\right)=5,7$ e 5,7 ; $\mathrm{pH}$ (índice $\mathrm{SMP})=5,9$ e 6,1 ; M.O.= 31 e $35 \mathrm{~g} /$ $\mathrm{dm}^{3} ; \mathrm{P}($ Mehlich $)=24,8$ e $22,4 \mathrm{mg} / \mathrm{dm}^{3}$; $\mathrm{H}+\mathrm{Al}=49,3$ e $40,7 \mathrm{mmol} / \mathrm{dm}^{3} ; \mathrm{K}=2,5 \mathrm{e}$ $4,6 \mathrm{mmolc} / \mathrm{dm}^{3} ; \mathrm{CTC}\left(\mathrm{pH}_{7,0}\right)=171$ e 169 $\mathrm{mmol}_{\mathrm{c}} / \mathrm{dm}^{3} ; \mathrm{Al}=0,0$ e $0,0 \mathrm{mmol} / \mathrm{dm}^{-3}$.

Antes da instalação dos experimentos, no início de janeiro de cada ano, a área experimental foi semeada com milheto (Pennisetum glaucum) e mucuna preta (Stizolobium aterrimum) na proporção de 30 e $40 \mathrm{~kg}$ de sementes/ha, respectivamente.

No transplantio das mudas adotou-se o sistema de cultivo mínimo, com revolvimento do solo apenas na linha de plantio, utilizando-se para isto, um microtrator adaptado com enxadas de corte sobre a palha das referidas plantas de cobertura. Essas, após completarem seu ciclo, foram acamadas com rolo-faca conforme as datas de transplante, momento em que também realizou-se as adubações de base nos tratamentos minerais e orgânicos. O espaçamento utilizado foi $40 \mathrm{~cm}$ entre linhas e 10 $\mathrm{cm}$ entre plantas. Não foi realizado o fornecimento de água (irrigação) após o transplante das mudas, contando-se apenas com a precipitação ocorrida nos períodos experimentais.

Para ambos os experimentos a adubação de base foi realizada com o equivalente a $120 \mathrm{~kg} / \mathrm{ha}$ de $\mathrm{P}_{2} \mathrm{O}_{5}$ e 90 $\mathrm{kg} / \mathrm{ha}$ de $\mathrm{K}_{2} \mathrm{O}$ para CONV, AM/CB e AM. As fontes utilizadas foram o superfosfato triplo (2011), superfosfato simples (2012) e cloreto de potássio (2011 e 2012). A adubação nitrogenada correspondeu à dose de $75 \mathrm{~kg} / \mathrm{ha}$ de $\mathrm{N}$, aplicado na forma de uréia (2011) ou nitrato de amônio (2012) no plantio e em três coberturas $(45,65$ e 90 dias após o transplante, DAT) correspondendo respectivamente a $20,35,25$ e $20 \%$ da dose total. As quantidades de fertilizantes minerais seguiram as recomendações da CQFSRS/SC (Comissão de Química e Fertilidade do Solo, 2004) tendo por base a análise de solo.

Nos tratamentos orgânicos, a adubação de base foi feita com $133 \mathrm{~g} / \mathrm{m}^{2}$ de fosfato natural reativo ( $9 \%$ ) para suprir a dose de $\mathrm{P}_{2} \mathrm{O}_{5}$ recomendada pela CQFSRS/SC (2004). Devido ao alto teor de potássio no solo, nenhuma fonte deste nutriente foi aplicada. Aos 30 DAT aplicou-se em cobertura, em linha e ao lado das plantas $0,64 \mathrm{~kg} / \mathrm{m}^{2}$ de esterco de peru, o equivalente a $75 \mathrm{~kg} / \mathrm{ha}$ de $\mathrm{N}$.

Os biofertilizantes utilizados nos experimentos foram produzidos tendo por base os materiais e proporções: $10 \mathrm{~kg}$ de esterco bovino fresco, $2 \mathrm{~kg}$ de casca de arroz incinerada; $2 \mathrm{~kg}$ de fosfato natural reativo (9\%), $2 \mathrm{~L}$ de leite (inoculante usado para acelerar a fermentação) e 30 L de água. Em 2011, o biofertilizante foi 
produzido de forma anaeróbica. Nesse processo, os materiais puros foram colocados em um recipiente, misturados e agitados uma vez ao dia, durante um mês. Em 2012, o biofertilizante utilizado foi produzido de forma anaeróbica conforme a metodologia proposta por Santos (1992). Quando prontos, os materiais resultantes de cada forma de preparo foram coados, armazenados em garrafas PET e encaminhados ao laboratório da Epagri Cepaf em Chapecó-SC, para análise como resíduo orgânico, de acordo com a metodologia proposta por Tedesco et al. (1995). A análise mineral dos biofertilizantes revelou, para os materiais produzidos de forma aeróbica e anaeróbica, respectivamente: $\mathrm{pH}$ em $\mathrm{CaCl}_{2}=7,3$ e 6,1; umidade $65^{\circ} \mathrm{C}=95,9$ e $98,6 \% ; \mathrm{N}=1,38$ e $0,88 \mathrm{~g} / \mathrm{L} ; \mathrm{P}_{2} \mathrm{O}_{5}=6,7$ e $0,8 \mathrm{~g} / \mathrm{L} ; \mathrm{K}_{2} \mathrm{O}=6,7$ e $1,4 \mathrm{~g} / \mathrm{L} ; \mathrm{Ca}=2,2$ e $0,3 \mathrm{~g} / \mathrm{L} ; \mathrm{Mg}=1,4$ e 0,3 g/L; em 2012 foi realizada, também, a análise de micronutrientes: $\mathrm{Cu}=1,4$ $\mathrm{mg} / \mathrm{L} ; \mathrm{Zn}=2,3 \mathrm{mg} / \mathrm{L} ; \mathrm{Fe}=35,85 \mathrm{mg} / \mathrm{L}$ e $\mathrm{Mn}=11,64 \mathrm{mg} / \mathrm{L}$.

O biofertilizante $(3,5$ ou $10 \%)$, a calda bordalesa $(0,3 \%)$ e o metalaxyl $-\mathrm{m}$ + mancozeb foram aplicados, de acordo com cada tratamento, ou seja, quinzenalmente, a partir de 30 dias após o transplante, totalizando seis e sete aplicações nos experimentos de 2011 e 2012, respectivamente. O manejo do tripes no tratamento CONV foi realizado com lambda-cialotrina (25 g i.a./ha). Esse princípio ativo foi pulverizado duas vezes em 2011 e três vezes em 2012, junto às últimas aplicações dos tratamentos fitossanitários.

Em 01/11/2011 (112 DAT) e 08/10/2012 (90 DAT), avaliaram-se o número de folhas e o índice de clorofila de três plantas aleatorizadas de cada parcela útil. As medições dos índices de clorofila foram realizadas, com auxílio de um clorofilômetro (Clorofilog-CFL1030 - Falker ${ }^{\circledR}$ ), na porção central da primeira folha totalmente expandida e de maior comprimento, em dia ensolarado.

Em 03/11/11 (114 DAT) e 30/10/12 (112 DAT) realizaram-se avaliações de tripes (Thrips tabaci), em cinco plantas por parcela em ambas as faces da planta de acordo com a simetria. Nesta avaliação utilizou-se a escala visual de notas, para determinar os danos causados por tripes pela raspagem das folhas e seca de pontei- ros. As notas foram atribuídas, respectivamente, com valores de 1, 3 e 9, para danos baixo, médio e alto. Nas mesmas datas e plantas também avaliaram-se os danos provocados por míldio, considerando a percentagem de área foliar necrosada.

As colheitas foram feitas quando as plantas apresentavam mais de $60 \%$ de tombamento.

No experimento de 2011, após a cura a campo (cinco dias após a colheita), foram retirados cinco bulbos por parcela útil experimental (equivalente a 0,22 $\mathrm{m}^{2}$ da área útil total de cada parcela experimental) para as avaliações de fitomassa seca dos bulbos (FSB), teor e exportação de nutrientes. A análise de FSB foi realizada individualmente para cada um dos cinco bulbos de cada parcela experimental. Para tal, o material foi acondicionado em sacos de papel e levado à estufa a $65^{\circ} \mathrm{C}$ até adquirir peso constante. Em seguida foi encaminhado para a análise de tecido, em laboratório da Embrapa Clima Temperado. A análise de exportação de nutrientes considerou a FSB média de cinco bulbos e a análise de tecido.

$\mathrm{Na}$ mesma ocasião foi realizada a coleta de bulbos, de uma área de 6,21 $\mathrm{m}^{2}$ da área útil total de cada parcela experimental, para as análises de produtividade. Os bulbos obtidos, após a retirada de raízes e talos das plantas, foram classificados de acordo com o diâmetro transversal (DT), conforme as normas estabelecidas para a cultura (Brasil, 1995). Após a classificação, os bulbos foram pesados com vistas à obtenção das fitomassas frescas por área (produtividade) de cada classe. As variáveis de produtividade consideradas foram produtividade comercial total (PCT-DT ${ }^{3}<35 \mathrm{~mm}$ ), de bulbos da classe 2 (C2-DT 35 a $50 \mathrm{~mm}$ ), de bulbos da classe 3 (C3-DT >50 a $70 \mathrm{~mm}$ ), e de bulbos da classe 3 e superiores (C3+- DT >50 mm).

Após a classificação e pesagem, retirou-se $1 \mathrm{~kg}$ de bulbos de cada parcela colhida para análise da qualidade nutricional, o restante foi ensacado e levado ao estaleiro, onde permaneceu por quatro meses. Ao fim desse período realizou-se a análise de conservação pós-colheita, considerando-se a percentagem de perda de fitomassa fresca inicial dos bulbos.

Para a análise da qualidade nutricional, determinação da composição mineral (P total, Fe, Se, Si, Ca, K e Na), amostras de bulbos foram encaminhadas à empresa Bioagri Alimentos e Ambiental, SP, que adotou o método (ICP-OES) POPPA 035/SMWW3120 USEPA 6010.

No ano de 2012 realizaram-se apenas as análises de FSB, produtividade e pós-colheita.

Os dados foram submetidos à análise de variância pelo teste $\mathrm{F}$ através dos programas estatísticos SANEST (Zonta \& Machado, 1984) e STATISTICA (STATSOFT, 2004). Ao alcançar a significância $(\mathrm{p}>0,05)$, as variáveis foram analisadas pelo teste de médias de Tukey. A análise de regressão linear múltipla com o uso do procedimento "stepwise" do programa SAS (1996) foi aplicada entre a relação da produtividade em 2011 com as variáveis, incidência de tripes, área necrosada por míldio e teores de nutrientes dos bulbos, e em 2012 com incidência de tripes e área necrosada por míldio.

\section{RESULTADOS E DISCUSSÃO}

Em 2011 aos 112 DAT, o índice de clorofila e número de folhas foi similar entre os tratamentos, sendo os valores médios situados em 64,83 e 10,42, respectivamente (Tabela 1). Em 2012, aos 90 DAT, maiores índices médios de clorofila $(80,20)$ e número de folhas $(10,57)$ foram observados em CONV, não diferindo dos demais tratamentos com adubação mineral. Pesquisas recentes mostram que o conteúdo de clorofila foliar está correlacionado à concentração de N na planta, assim como o aumento do fornecimento de nitrogênio proporciona a formação de maior número de folhas na cultura da cebola (Vidigal \& Moreira, 2009; Kurtz et al., 2012).

Os resultados de 2011 indicam que, independente do manejo adotado, as plantas apresentaram os mesmos índices de clorofila e estádio de desenvolvimento e, indiretamente, indicam que o fornecimento de nitrogênio foi similar para todos os tratamentos. Convém ressaltar que em 2011 as medições foram realizadas aos 112 DAT, ou seja, 
Tabela 1. Índice de clorofila (IC), número de folhas (NF) e fitomassa seca dos bulbos (FSB) de acordo com os sistemas de cultivo adotados para a cultivar Empasc 352 Bola Precoce [chlorophyll index (IC), leaf number (NF) and dry phytomass of bulbs (FSB) according to the adopted cropping systems for the cultivar Empasc 352 Bola Precoce]. Ituporanga, Epagri, 2012.

\begin{tabular}{|c|c|c|c|c|c|c|}
\hline \multirow{2}{*}{ Tratamento* } & \multicolumn{2}{|c|}{ IC } & \multicolumn{2}{|c|}{ NF } & \multicolumn{2}{|c|}{ FSB (g/bulbo) } \\
\hline & 2011 & 2012 & 2011 & 2012 & 2011 & 2012 \\
\hline $\mathrm{CONV}$ & $69,16 \mathrm{~ns}$ & $80,20 \mathrm{a}$ & $9,67 \mathrm{~ns}$ & $10,57 \mathrm{a}$ & $12,85^{\mathrm{ns}}$ & $13,89 \mathrm{a}$ \\
\hline $\mathrm{AM}$ & - & $76,03 \mathrm{ab}$ & - & $9,07 \mathrm{~b}$ & - & $6,79 \mathrm{~b}$ \\
\hline $\mathrm{AM} / \mathrm{CB}$ & 63,83 & $77,60 \mathrm{ab}$ & 8,42 & $8,50 \mathrm{~b}$ & 10,29 & $7,26 \mathrm{~b}$ \\
\hline $\mathrm{AO}$ & 65,63 & $73,26 \mathrm{~b}$ & 8,16 & $8,42 \mathrm{~b}$ & 10,75 & $6,43 \mathrm{~b}$ \\
\hline $\mathrm{AO} / \mathrm{CB}$ & 62,00 & $73,14 \mathrm{~b}$ & 8,83 & $8,32 \mathrm{~b}$ & 9,55 & $6,64 \mathrm{~b}$ \\
\hline $\mathrm{AO} / \mathrm{CB} / \mathrm{BF}-3 \%$ & 66,03 & $73,41 \mathrm{~b}$ & 8,41 & $8,15 \mathrm{~b}$ & 9,39 & $6,58 \mathrm{~b}$ \\
\hline $\mathrm{AO} / \mathrm{CB} / \mathrm{BF}-5 \%$ & 68,70 & $77,95 \mathrm{ab}$ & 8,83 & $8,00 \mathrm{~b}$ & 10,23 & $6,95 \mathrm{~b}$ \\
\hline $\mathrm{AO} / \mathrm{CB} / \mathrm{BF}-10 \%$ & 58,43 & $73,47 \mathrm{~b}$ & 8,41 & $8,00 \mathrm{~b}$ & 9,32 & $6,57 \mathrm{~b}$ \\
\hline CV (\%) & 10,93 & 3,41 & 0,45 & 0,19 & 15,66 & 8,15 \\
\hline
\end{tabular}

Médias seguidas de mesmas letras na coluna não diferem entre si, Tukey a 1 ou 5\%; *CONV=adubação mineral e agrotóxicos convencionais; $\mathrm{AM}=$ adubação mineral; $\mathrm{CB}=$ calda bordalesa $0,3 \% ; \mathrm{AO}=$ adubação orgânica; $\mathrm{BF}=$ biofertilizante líquido à base de esterco bovino a 3,5 ou $10 \%$ (average values followed by same letters in the column do not differ from each other, Tukey, 1 or $5 \% ; * \mathrm{CONV}=$ conventional mineral fertilizer and pesticides; $\mathrm{AM}=$ mineral fertilizer without pest management; $\mathrm{CB}=$ Bordeaux mixture $0.3 \%$; $\mathrm{AO}=$ organic fertilizer; $\mathrm{BF}=$ biofertilizer of cow manure, 3,5 or $10 \%)$.

Tabela 2. Produtividade comercial total (PCT), de bulbos da classe 2 (C2), classe 3 (C3) e da classe 3 e superiores (C3+) de acordo com os sistemas de cultivo adotados para a cultivar Empasc 352 Bola Precoce [total marketable (PCT), class 2 (C2), class 3 (C3), class 3 and superior $(\mathrm{C} 3+)$ productivities according to the cropping systems adopted for the cultivar Empasc 352 Bola Precoce]. Ituporanga, Epagri, 2012.

\begin{tabular}{|c|c|c|c|c|c|c|c|c|}
\hline \multirow{2}{*}{ Tratamento* } & \multicolumn{2}{|c|}{ PCT (t/ha) } & \multicolumn{2}{|c|}{ C2 (t/ha) } & \multicolumn{2}{|c|}{ C3 (t/ha) } & \multicolumn{2}{|c|}{ C3+ (t/ha) } \\
\hline & 2011 & 2012 & 2011 & 2012 & 2011 & 2012 & 2011 & 2012 \\
\hline $\mathrm{CONV}$ & $31,09 \mathrm{a}$ & $20,62 \mathrm{a}$ & $0,17 \mathrm{~b}$ & $0,17 \mathrm{c}$ & $21,24^{\mathrm{ns}}$ & $14,77 \mathrm{a}$ & $30,92 \mathrm{a}$ & $20,45 \mathrm{a}$ \\
\hline $\mathrm{AM}$ & - & $8,54 \mathrm{c}$ & - & $0,55 \mathrm{~b}$ & - & $3,02 \mathrm{~b}$ & - & $7,99 \mathrm{~b}$ \\
\hline $\mathrm{AM} / \mathrm{CB}$ & $22,38 \mathrm{~b}$ & $9,54 \mathrm{bc}$ & $0,35 \mathrm{ab}$ & $0,59 \mathrm{~b}$ & 18,54 & $4,38 \mathrm{~b}$ & $22,03 \mathrm{~b}$ & $8,88 \mathrm{~b}$ \\
\hline $\mathrm{AO}$ & $19,65 \mathrm{~b}$ & $11,27 \mathrm{bc}$ & $0,40 \mathrm{a}$ & $0,40 \mathrm{a}$ & 15,64 & $1,94 \mathrm{~b}$ & $19,25 \mathrm{~b}$ & $10,34 \mathrm{~b}$ \\
\hline $\mathrm{AO} / \mathrm{CB}$ & $20,41 \mathrm{~b}$ & $11,39 \mathrm{bc}$ & $0,35 \mathrm{ab}$ & $0,90 \mathrm{a}$ & 16,93 & $2,34 \mathrm{~b}$ & $20,06 \mathrm{~b}$ & $10,48 \mathrm{~b}$ \\
\hline $\mathrm{AO} / \mathrm{CB} / \mathrm{BF}-3 \%$ & $20,19 b$ & $11,44 b c$ & $0,36 \mathrm{ab}$ & $0,80 \mathrm{a}$ & 16,63 & $4,23 \mathrm{~b}$ & $19,83 \mathrm{~b}$ & $10,64 b$ \\
\hline $\mathrm{AO} / \mathrm{CB} / \mathrm{BF}-5 \%$ & $19,99 \mathrm{~b}$ & $12,13 \mathrm{~b}$ & $0,37 \mathrm{a}$ & $0,86 \mathrm{a}$ & 16,21 & $3,52 \mathrm{~b}$ & $19,62 \mathrm{~b}$ & $11,27 b$ \\
\hline $\mathrm{AO} / \mathrm{CB} / \mathrm{BF}-10 \%$ & $19,40 \mathrm{~b}$ & $10,94 \mathrm{bc}$ & $0,39 \mathrm{a}$ & $0,87 \mathrm{a}$ & 15,47 & $2,29 \mathrm{~b}$ & $19,01 \mathrm{~b}$ & $9,82 \mathrm{~b}$ \\
\hline $\mathrm{CV}(\%)$ & 13,5 & 9,88 & 31,2 & 9,76 & 18,7 & 26,9 & 14,1 & 10,5 \\
\hline
\end{tabular}

Médias seguidas de mesma letra na coluna não diferem entre si, Tukey, $1 \%$; ${ }^{*} \mathrm{CONV}=$ adubação mineral e agrotóxicos convencionais; $\mathrm{AM}=$ adubação mineral; $\mathrm{CB}=$ calda bordalesa $0,3 \% ; \mathrm{AO}=$ adubação orgânica; $\mathrm{BF}=$ biofertilizante líquido à base de esterco bovino a 3,5 ou $10 \%$ (average values followed by same letters in the column do not differ from each other, Tukey, $1 \%$; ${ }^{*} \mathrm{CONV}=$ conventional mineral fertilizer and pesticides; $\mathrm{AM}=$ mineral fertilizer without pest management; $\mathrm{CB}=$ Bordeaux mixture $0.3 \%$; $\mathrm{AO}=$ organic fertilizer; $\mathrm{BF}=$ liquid biofertilizer of cow manure, 3,5 or $10 \%)$.

próximas ao final do ciclo da cultura e, por conseguinte, no momento em que os teores de nitrogênio tendem a decrescer, o que pode ter uniformizado os índices de clorofila observados. Em 2012, aos 90 DAT, os maiores índices de clorofila e número de folhas foram observados nos tratamentos com adubação mineral (Tabela 1), em relação aos tratamentos com adubação orgânica e com o uso de biofertilizante. Isto pode ter ocorrido devido ao menor teor de nitrogênio presente no biofertilizante utilizado na pulverização das plantas.

Ao comparar os anos agrícolas, observa-se que em 2012 (Tabela 1), aos 90 DAT, foram registrados índices de clorofila maiores que em 2011. Isso se deve, como anteriormente mencionado, ao momento em que as medições foram realizadas. Outro fator que pode ter contribuído para a elevação dos índices de clorofila em 2012 pode ter sido a ocorrência de déficit hídrico, uma vez que nesse ano o volume de chuvas foi metade do registrado em 2011. Estudos realizados por Carvalho et al. (2003) revelam que plantas de artemísia (Tanacetum parthenium) cultivadas em vasos, mantidas a $50 \%$ da capacidade de campo mostraram, durante todo o período de cultivo, índices de clorofila cerca de $30 \%$ mais altos em relação a plantas mantidas a $90 \%$ da capacidade de campo.

No ensaio de 2011, a fitomassa seca dos bulbos (FSB) não diferiu entre tratamentos, com valor médio de 10,34 g (Tabela 1). Em 2012, ao contrário, as maiores quantidades de FSB foram registradas para sistema de manejo 
convencional $(13,89 \mathrm{~g})$. Nesse ano, a FSB média dos tratamentos foi de 7,64 g. Os menores valores médios de FSM em 2012 foram devidos à ocorrência de déficit hídrico.

Em 2011 também não houve diferença significativa $(\mathrm{p}>0,05)$ em relação à qualidade nutricional, teores e exportação de nutrientes dos bulbos, à exceção do Mn. Nesse caso, a maior exportação de Mn foi observada no tratamento CONV (73,1 g/ha), enquanto os demais retiraram, em média, do sistema $46,8 \mathrm{~g} / \mathrm{ha}(\mathrm{CV}=17,2 \%)$ do elemento. A maior exportação de $\mathrm{Mn}$ pelo tratamento CONV está associada à aplicação do fungicida metalaxyl-m + mancozeb, o qual possui o nutriente em sua formulação.

Em relação à qualidade nutricional, os dados de composição médios da cebola brasileira crua segundo a TACO (2006) convertidos para massa seca são de $15,9 \mathrm{~g} / \mathrm{kg}$ de $\mathrm{K}, 3,4 \mathrm{~g} / \mathrm{kg}$ de $\mathrm{P}, 1,3 \mathrm{~g} / \mathrm{kg}$ de $\mathrm{Ca}, 90,1 \mathrm{mg} / \mathrm{kg}$ de $\mathrm{Na}$ e $18,0 \mathrm{mg} / \mathrm{kg}$ de Fe. A análise da composição mineral dos bulbos no presente trabalho revelou valores médios de $16,5 \mathrm{~g} / \mathrm{kg}$ de $\mathrm{K}(\mathrm{CV}=$ $7,5 \%), 3,4 \mathrm{~g} / \mathrm{kg}$ de $\mathrm{P}(\mathrm{CV}=10,7 \%), 1,4$ $\mathrm{g} / \mathrm{kg}$ de $\mathrm{Ca}(\mathrm{CV}=16,8 \%), 210,0 \mathrm{mg} / \mathrm{kg}$ de $\mathrm{Na}(\mathrm{CV}=30,2 \%)$ e $63,0 \mathrm{mg} / \mathrm{kg}$ de $\mathrm{Fe}(\mathrm{CV}=6,6 \%)$. Portanto, independente do sistema de produção, mineral ou orgânico, os níveis dos nutrientes foram próximos ou superiores aos observados na TACO (2006). Os teores médios de $\mathrm{Si}$ e $\mathrm{Se}$ dos bulbos nos tratamentos do ensaio de 2011 estiveram, respectivamente, abaixo de 125,0 e $<0,25 \mathrm{mg} / \mathrm{kg}$. Os resultados para os elementos foram superiores para $\mathrm{K}$ e Fe, e inferiores para $\mathrm{P}, \mathrm{Ca}, \mathrm{Na}, \mathrm{Si}$ e $\mathrm{Se}$ aos observados por Gonçalves et al. (2011, 2012) em cebola cultivada em sistema orgânico em propriedades do Alto Vale do Itajaí-SC, e na Epagri, Estação Experimental de Ituporanga.

Os teores médios de macronutrientes, análise de tecido dos bulbos, foram de: $13,5 \mathrm{~g} / \mathrm{kg}$ de $\mathrm{N}(\mathrm{CV}=5,5 \%) ; 5,3$ $\mathrm{g} / \mathrm{kg}$ de $\mathrm{P}(\mathrm{CV}=16,7 \%) ; 13,1 \mathrm{~g} / \mathrm{kg}$ de $\mathrm{K}(\mathrm{CV}=3,3 \%) ; 3,8 \mathrm{~g} / \mathrm{kg}$ de $\mathrm{Ca}(\mathrm{CV}=$ $7,6 \%)$ e $1,3 \mathrm{~g} / \mathrm{kg}$ de $\mathrm{Mg}(\mathrm{CV}=4,4 \%)$; enquanto os valores médios de micronutrientes se situaram em: 142,0 mg/ $\mathrm{kg}$ de $\mathrm{Fe}(\mathrm{CV}=27,3 \%) ; 19,4 \mathrm{mg} / \mathrm{kg}$ de $\mathrm{Mn}(\mathrm{CV}=10.8 \%) ; 13,5 \mathrm{mg} / \mathrm{kg}$ de $\mathrm{Zn}$
$(\mathrm{CV}=12,3 \%) ; 4,8 \mathrm{mg} / \mathrm{kg}$ de $\mathrm{Cu}(\mathrm{CV}=$ $17,0 \%)$ e $11,6 \mathrm{mg} / \mathrm{kg}$ de $\mathrm{B}(\mathrm{CV}=15,2 \%)$. Observa-se que os teores de nutrientes seguiram, em ordem decrescente, $\mathrm{N}>\mathrm{K}>\mathrm{P}>\mathrm{Ca}>\mathrm{Mg}$ para macronutrientes e $\mathrm{Fe}>\mathrm{Mn}>\mathrm{Zn}>\mathrm{B}>\mathrm{Cu}$ para micronutrientes.

A exportação de nutrientes pelos bulbos foi em média de: $35,3 \mathrm{~kg} /$ ha de $\mathrm{N}(\mathrm{CV}=21,7 \%) ; 14,0 \mathrm{~kg} / \mathrm{ha}$ de $\mathrm{P}(\mathrm{CV}=$ $36,3 \%) ; 33,8 \mathrm{~kg} /$ ha de $\mathrm{K}(\mathrm{CV}=19,1 \%)$; $9,7 \mathrm{~kg} / \mathrm{ha}$ de $\mathrm{Ca}(\mathrm{CV}=18,1 \%) ; 3,4 \mathrm{~kg} /$ ha de $\mathrm{Mg}(\mathrm{CV}=19,7 \%) ; 371,7 \mathrm{~g} /$ ha de $\mathrm{Fe}(\mathrm{CV}=31,7 \%) ; 35,2 \mathrm{~g} / \mathrm{ha}$ de $\mathrm{Zn}(\mathrm{CV}=$ $22,6 \%) ; 12,6 \mathrm{~g} / \mathrm{ha}$ de $\mathrm{Cu}(\mathrm{CV}=26,3 \%)$; e $0,03 \mathrm{~g} / \mathrm{ha}$ de $\mathrm{B}(\mathrm{CV}=23,6 \%)$. A ordem de exportação de macronutrientes pelos bulbos da cultivar Bola Precoce $(\mathrm{N}>\mathrm{K}>\mathrm{P}>\mathrm{Ca}>\mathrm{Mg})$ é discordante da observada por Pôrto et al. (2006) para o híbrido Optima $(\mathrm{K}>\mathrm{N}>\mathrm{Ca}>\mathrm{Mg}>\mathrm{P})$ e por Santos et al. (2007) para as cultivares Franciscana IPA10 e Alfa São Francisco $(\mathrm{N}>\mathrm{K}>\mathrm{Ca}>\mathrm{P}>\mathrm{Mg})$. As diferenças observadas podem ser atribuídas à exigência nutricional de cada cultivar, classe de solo, sistema de cultivo, produtividade e ciclo cultural (Vidigal et al., 2003), e evidenciam a necessidade de estudos específicos para cada cultivar, condição edafoclimáticas e sistema de produção adotado.

Nos dois ensaios, a produtividade comercial total (PCT) (soma das classes 2 e $\mathrm{C} 3+$ ) e de bulbos da classe C3+ (soma da classe 3 e de bulbos de diâmetro superior a $70 \mathrm{~mm}$ ) diferiu significativamente entre o tratamento convencional (CONV) e os demais. No CONV foram registradas a formação de bulbos de maior diâmetro médio transversal e as mais altas produtividades comerciais (Tabela 2).

Em 2011, não houve diferenças significativas entre os tratamentos para os bulbos da classe 3 (C3), cuja produtividade média foi de $17,24 \mathrm{t} / \mathrm{ha}$ (Tabela 2). Tal fato pode ser explicado pelo manejo de solo, uso de plantas de cobertura/adubação verde, aplicado em todos os tratamentos. Gonçalves et al. (2004) em sistema de cultivo orgânico de cebola sob plantas de cobertura (mucuna e milho), adubação com esterco suíno (equivalente a $37,5 \mathrm{~kg} /$ ha de $\mathrm{N}$ ) e uso de biofertilizantes de esterco bovino obtiveram produtividades similares às observadas no presente trabalho, de 17,8 a 20,2 t/ha, sendo a produtividade da testemunha (sem aplicação de biofertilizante) de 20,7 t/ha. Isto sugere que as adubações de base e manejos de solo com plantas de cobertura/adubação verde podem atuar como fatores homogeneizadores da produtividade, explicando, dessa forma, as produtividades semelhantes entre os tratamentos, obtidas na safra de 2011. A ausência de diferenças significativas $(\mathrm{p}<0,05)$ entre os tratamentos relacionados ao teor e exportação de nutrientes pelos bulbos indicam a veracidade de tal hipótese. A produtividade obtida em ano de precipitação normal, 2011, foi superior ao constatado em propriedades rurais do Alto vale do Itajaí-SC, sob sistema orgânico por Gonçalves \& Wamser (2007), 11 a 15 t/ha. A PCT obtida no tratamento CONV foi similar à obtida por Wordell Filho et al. (2007), 31,2 t/ha, e por Kurtz \& Ernani (2010) sem a aplicação de micronutrientes no solo, 19,5 a 32,2 2 t/ha, em sistema convencional, em solo similar ao do presente trabalho.

Nos experimentos, observa-se que a menor formação de bulbos da classe 2 (C2) foi obtida no tratamento convencional (CONV), enquanto os maiores valores foram observados, principalmente, nos sistemas orgânicos (Tabela 2). Tal comportamento em 2011, possivelmente, está associado a distúrbios nutricionais provocados pela adição de biofertilizantes em maiores concentrações, uma vez que, quando o biofertilizante foi aplicado a 3\%, não houve diferença significativa de produtividade para $\mathrm{C} 2$ em relação ao tratamento convencional. Por sua vez, em 2012, as repostas obtidas estão associadas ao déficit hídrico.

A baixa disponibilidade de água no solo induz a planta ao fechamento estomático. Quando isso ocorre, tanto a fotossíntese quanto o fluxo de nutrientes do solo para as raízes, seja por fluxo de massa ou difusão, são diminuídos (Larcher, 2000; Taiz \& Zeiger, 2006; Ernani, 2008). Portanto, é de se esperar que a ocorrência de déficits hídricos durante o cultivo, notadamente no período de bulbificação, dificulte o desenvolvimento normal das plantas, promova a formação de bulbos menores e, em con- 
sequência, reduza significativamente a produtividade (Wordell Filho \& Stadnik, 2010). Desse modo, a menor produtividade obtida em 2012 está relacionada à condição de baixa pluviosidade, em especial quando ela ocorre no período inicial da formação dos bulbos. O sistema de produção convencional (CONV) se destacou como aquele em que foram obtidas as maiores produtividades comerciais e a menor formação de bulbos de menor diâmetro médio transversal, o que possivelmente se deve à melhor nutrição e controle fitossanitário.

Em relação ao último aspecto, é possível que as menores PCT observadas nos tratamentos não convencionais estejam relacionadas à incidência de míldio durante o ciclo de cultivo. Em trabalho desenvolvido por Wordell Filho et al. (2007), os autores observaram que a redução da severidade do míldio, em 60 ou $23 \%$, somente é conseguida pela pulverização semanal de fungicidas sintéticos ou fertilizantes $(400 \mathrm{~mL}$ p.c. 100 L; 03-00-16, respectivamente), quando comparada à testemunha não pulverizada. No presente experimento a diferença da PCT entre o tratamento convencional e os demais foi em média de 35 e $48 \%$, em ano sem (2011) e com (2012) déficit hídrico, respectivamente. Nesse sentido, verifica-se que uma única análise da doença durante o ciclo da cultura se mostra insuficiente para relacioná-la à produtividade. Isso explica a ausência de diferenças significativas entre os tratamentos para a incidência de míldio observada nos ensaios conduzidos em 2011 e 2012.

Nos dois ensaios, o teste de médias relativo às análises da incidência de tripes (IT) e percentagem de folhas necrosadas por míldio (IM) não indicou diferenças significativas entre os tratamentos. No entanto, em 2011, o modelo de regressão linear múltipla mostrou que existem relações entre a produtividade comercial total (PCT) e as variáveis: teores de nutrientes, IT e IM, sendo obtida a seguinte equação, $\mathrm{PCT}=10,57-3,87 \mathrm{IT}-0,62 \mathrm{IM}+21,00 \mathrm{~N}$ $+0,05 \mathrm{Fe}, \mathrm{R}^{2}=0,68 \%$. Portanto, a PCT esteve positivamente relacionada ao teor de nitrogênio $\left(\mathrm{R}^{2}=0,27\right)$ e de ferro $\left(\mathrm{R}^{2}=\right.$ $0,16)$, e negativamente à incidência de tripes $\left(\mathrm{R}^{2}=0,15\right)$ e míldio $\left(\mathrm{R}^{2}=0,10\right)$.
Em relação à produtividade de bulbos menores, classe 2 (C2), a equação gerada pelo modelo foi $\mathrm{C} 2=0,27+0,20$ IT $-0,30 \mathrm{~N}+0,67 \mathrm{Ca}-0,001 \mathrm{Fe}-0,05 \mathrm{Cu}$ $(\mathrm{R} 2=0,67)$. Desta maneira, foi observado que a C2 esteve relacionada à $\mathrm{IT}\left(\mathrm{R}^{2}=\right.$ $0,30)$, seguida pelo teor de cobre $\left(\mathrm{R}^{2}=\right.$ $0,13)$, ferro $\left(R^{2}=0,09\right)$, cálcio $\left(R^{2}=0,09\right)$ e $\mathrm{N}\left(\mathrm{R}^{2}=0,06\right)$. Portanto, o aumento na formação de bulbos da classe 2 esteve associado, principalmente, à incidência de tripes.

A perda de fitomassa de bulbos após quatro meses de armazenamento não diferiu entre os tratamentos sendo em média de $23,9 \%(\mathrm{CV}=13,64 \%)$ e $32,4 \%(\mathrm{CV}=$ 19,59\%) em 2011 e 2012, respectivamente. Portanto, os manejos de adubação e fitossanitários aplicados não influenciaram a conservação dos bulbos em pós-colheita.

Os ensaios revelaram que: o sistema de cultivo mínimo convencional foi mais produtivo em relação ao orgânico; as medições indiretas de clorofila são influenciadas pela fase do ciclo e podem ser afetadas pela ocorrência de estresse hídrico e; déficits hídricos são capazes de reduzir a produtividade comercial total da cultura em 34\% nos sistemas de cultivo mínimo convencional e, em média, 43\% em cultivos orgânicos. Em sistema orgânico de produção de cebola o uso de biofertilizante à base de esterco bovino nas doses, concentrações e parcelamentos efetuados não forneceu nutrientes, em especial nitrogênio, nas quantidades requeridas pelas plantas; não alterou a qualidade nutricional, teor e extração de nutrientes pelos bulbos; e não reduziu a incidência de tripes e míldio na cultura da cebola.

A ausência de respostas positivas à pulverização de biofertilizantes indica que estudos na cultura da cebola em sistemas orgânicos devem ser direcionados à fertilização do solo pelo uso de plantas de cobertura/adubação verde, associados à adubação de base e cobertura parcelada com materiais orgânicos (sólidos ou líquidos) sobre o solo, com estercos curtidos ou compostos, visando adequar o fornecimento de nutrientes à demanda das plantas e, desta forma, tornando-as mais resistentes a pragas e doenças e, por conseguinte, mais produtivas.

\section{AGRADECIMENTOS}

Os autores agradecem à FAPESC pelo financiamento da pesquisa, e ao valoroso auxílio dos colegas da Epagri Eng. Agr. Dr. Evandro Spagnollo, responsável pelo Laboratório de Análise do Solo da Epagri-EE de Chapecó, ao Téc. Agr. Marcelo Pitz e Assistente de Campo Odair Justen da Epagri-EE de Ituporanga.

\section{REFERÊNCIAS}

BETTIOL W. 2001. Resultados de pesquisa com métodos alternativos para o controle de doenças de plantas. In: ENCONTRO DE PROCESSOS DE PROTEÇÃO DE PLANTAS: CONTROLE ECOLÓGICO DE PRAGAS E DOENÇAS, 1. Resumos... Botucatu: Agroecológica. p. 125-135.

BETTONI MM. 2011. Desempenho de cultivares de cebola em sistema orgânico na região metropolitana de Curitiba. Curitiba: UFP Setor de Ciências Agrárias. 72p (Dissertação mestrado).

BRASIL. 1995. Ministério da Agricultura, Abastecimento e Reforma Agrária. Portaria n.529 de 18 ago. 1995. Diário Oficial da República Federativa do Brasil., Brasília, 1 de set., Seção 1:13513.

CARVALHO LM; CASALI VWD; SOUZA MA; CECON PR. 2003. Disponibilidade de água no solo e crescimento de artemísia. Horticultura Brasileira 21: 726-730.

COMISSÃO DE QUÍMICA E FERTILIDADE DO SOLO RS/SC - CQFS-RS/SC. 2004. Manual de adubação e de calagem para o Estado do Rio Grande do Sul e Santa Catarina. Porto Alegre: UFRGS. 400p.

EPAGRI. 2000. Sistema de produção para a cebola - Santa Catarina. Florianópolis: Epagri. 91p. (Sistemas de Produção n 16).

ERNANI PR. 2008. Química do solo e disponibilidade de nutrientes. Lages: CAV/ UDESC. 230p.

GONÇALVES PAS; VIEIRA NETO J; CARVALHO PGB. 2012. Efeito da pulverização foliar de preparados homeopáticos de Natrum muriaticum e calcário de conchas sobre a composição mineral de bulbos de cebola em sistema orgânico. Revista Agropecuária Catarinense 25: 80-84.

GONÇALVES PAS; VIEIRANETO J; LINDNER GH; VISENTAINER KM. 2011. Assessoria na avaliação da qualidade nutricional de bulbos de cebola produzida por agricultores orgânicos do Alto Vale do Itajaí, SC. In: CONGRESSO BRASILEIRO DE AGROECOLOGIA, 7. Resumos... Fortaleza: Cadernos de Agroecologia. p.5.

GONÇALVES PAS; WAMSER GH. 2007. Produção orgânica de cebola com agricultores familiares. Revista Brasileira de Agroecologia, 2: 63-68.

GONÇALVES PAS; WERNER H; DEBARBA JF. 
2004. Avaliação de biofertilizantes, extratos vegetais e diferentes substâncias alternativas no manejo de tripes em cebola em sistema orgânico. Horticultura Brasileira 22: 659-662.

IBGE - Instituto Brasileiro de Geografia e Estatística. 2010. 15 de setembro. Sistema $I B G E$ de recuperação automática - SIDRA. Disponível em http://www.sidra.ibge.gov.br

KURTZ C; ERNANI PR. 2010 Produtividade de cebola influenciada pela aplicação de micronutrientes. Revista Brasileira de Ciência do Solo 34: 133-142.

KURTZ C; ERNANI PR; COIMBRA JLM; PETRY E. 2012. Rendimento e conservação de cebola alterados pela dose e parcelamento de nitrogênio em cobertura. Revista Brasileira de Ciência do Solo 36: 865-875.

LARCHER W. 2000. Ecofisiologia vegetal. São Carlos: RiMa. 531p.

PÔRTO DRQ; CECÍLIO FILHO AB; MAY A; BARBOSA JC. 2006. Acúmulo de macronutrientes pela cultivar de cebola "Optima" estabelecida por semeadura direta. Horticultura Brasileira 24: 470-475.

SANTOS ACV. 2001. Biofertilizante líquido: o defensivo agrícola da natureza. 2 ed. rev. Niterói: EMATER-Rio.16p.
SANTOS ACV. 1992. Biofertilizante líquido $O$ defensivo agrícola da natureza. Niterói: EMATER-RIO, 16p. (Agropecuária Fluminense, 8).

SANTOS EEF; FERNANDES DM; SILVA DJ; BULL LT. 2007. Acúmulo de macronutrientes por cultivares de cebola, em um vertissolo no médio São Francisco. In: CONGRESSO BRASILEIRO DE CIÊNCIA DO SOLO, 31, 2007, Gramado. Anais... Porto Alegre: SBCS (CD-ROM).

SAS INSTITUTE. 1996. SAS, versão 6.12. Cary, NC: SAS Institute INC. CD-ROM.

SOUZA RB; ALCÂNTARA FA. 2008. Adubação no sistema orgânico de produção de hortaliças. Brasília: Embrapa Hortaliças. 8p. (Circular Técnica, 65).

STATSOFT, Inc. 2004. STATISTICA (data analysis software system), version 7. Disponível em http//www.statsoft.com

TACO. Tabela brasileira de composição de alimentos. 2006. 08 de abril de 2011. NEPAUNICAMP. Versão II. 2. ed. Campinas: NEPA-UNICAMP. Disponível em http://www. unicamp.br/nepa

TAIZ L; ZEIGER E. 2006. Fisiologia vegetal. 3.ed. Porto Alegre: Artmed. 722p.
TEDESCO MJ; GIANELLO C; BISSANI CA; BOHNEN H; VOLKWEISS SJ. 1995. Análises de solo, plantas e outros materiais. Porto Alegre: UFRGS. 188p.

VIDIGALSM; MOREIRAMA. 2009. Diagnóstico de nitrogênio por medidores portáteis para uso na cultura da cebola. Belo Horizonte: Epamig. 5p. (Circular Técnica, 52).

VIDIGAL SM; PEREIRA PRG; PACHECO DD; FACION CE. 2003. Acumulação de matéria fresca e seca pela cebola. In: CONGRESSO BRASILEIRO DE OLERICULTURA, 43. Resumos... Recife: SOB (CD-ROM).

WORDELL FILHO JA; MARTINS DA; STADNIK MJ. 2007. Aplicação foliar de tratamentos para o controle do míldio e da podridão-de-escamas de bulbos de cebola. Horticultura Brasileira 25: 544-549.

WORDELL FILHO JA; STADNIK MJ. 2010. Efeito de fosfitos de potássio e de manganês sobre o míldio da cebola. Agropecuária Catarinense 23: 84-87.

ZONTA EP; MACHADO AA. 1984. SANEST - Sistema de Análise Estatística para Microcomputadores. Pelotas: UFPel. 75 p. 